HT2009-88440

\title{
HEAT TRANSFER IN ADJACENT INTERACTING IMPINGING SYNTHETIC JETS
}

\author{
Tim Persoons \\ Mechanical Engineering dept., \\ Parsons Building, Trinity College \\ Dublin 2, Ireland \\ tim.persoons@tcd.ie
}

\author{
Tadhg S. O'Donovan \\ Mechanical Engineering dept., \\ Heriot-Watt University, \\ Edinburgh EH14 4AS, UK
}

\author{
Darina B. Murray \\ Mechanical Engineering dept., \\ Parsons Building, Trinity College \\ Dublin 2, Ireland
}

\section{ABSTRACT}

An impinging synthetic jet can attain heat transfer rates comparable to a continuous jet, without net mass input. However it needs a forced cross-flow to supply fresh cooling medium. The vectoring effect of adjacent synthetic jets allows directing the flow by changing the phase between the jets. This study uses the vectoring effect of two adjacent synthetic jets to draw in fresh air, while maintaining high impingement cooling performance.

The experimental approach applies infrared thermography and particle image velocimetry to quantify the local convective heat transfer and flow field, respectively. The heat transfer profiles for various phase differences have been compared to the mean flow field and wall-normal velocity fluctuation intensity.

For a fixed operating point (stroke length and Reynolds number) and geometry, the cooling performance has been optimised for phase and jet-to-surface spacing, resulting in about $90 \%$ enhancement of the maximum and overall cooling rate compared to a single jet, without the need for external cross-flow forcing.

Keywords: synthetic jet, impinging jet, vectoring, crossflow, electronics cooling, particle image velocimetry.

\section{INTRODUCTION}

Impinging synthetic jets have been identified as a promising technique for cooling miniature surfaces such as electronic packages [1-3]. A synthetic jet comprises a train of vortices formed by successive ejection and suction of fluid across an orifice. For a given orifice, the flow field of a free synthetic jet is governed by the Reynolds number $R e=\rho U_{0} D / \mu$ ( $D$ is a characteristic geometric length: the slot width) and stroke length $L_{0}$, based on the average ejection velocity $U_{0}$ :

$$
L_{0}=\int_{0}^{1 /(2 f)} U_{m}(t) \mathrm{d} t=2 U_{0} / f
$$

where $U_{m}(t)$ is the area-averaged orifice velocity and $f$ is the driving frequency. The flow field of free synthetic jets is wellknown [4]. An impinging synthetic jet is further characterised by its jet-to-surface spacing $H / D$.

For a single round impinging synthetic jet, a critical stroke length of $L_{0} / H \cong 2.5$ is found [5], marking two flow regimes: At low $L_{0} / H$, the vortices develop and lose strength before impingement. At high $L_{0} / H$, the flow tends to an intermittent on/off flow resulting in a time-averaged recirculation vortex. The two flow regimes are mirrored in two heat transfer regimes. At low $L_{0} / H$, the stagnation heat transfer increases with $L_{0}$. At high $L_{0} / H$, the stagnation heat transfer becomes independent of stroke length and can be approximated by $N u_{0}=1.75 \operatorname{Re}^{0.32} \operatorname{Pr}^{0.4}$ $\left(1<L_{0} / D<22,1000<R e<4300, H / D=2, P r=0.71\right)$ [5].

When a single impinging synthetic jet cools a heated surface, a forced cross-flow is required to supply fresh cooling medium. However, Smith and Glezer [6] investigated the flow field of a pair of adjacent synthetic jets. Vortex interaction induces a vectoring effect on the far field flow depending on the phase difference between the actuators. The flow is vectored towards the side of the jet leading in phase.

The current study applies this vectoring effect in a pair of impinging synthetic jets, thereby combining (i) high local heat transfer rates and (ii) the cross-flow required for effective cooling. The paper aims to determine the optimal conditions for convective cooling as a function of jet-to-surface spacing and phase difference between the jets.

\section{EXPERIMENTAL APPROACH}

\subsection{Test Rig}

Figure 1 shows a schematic of the test rig. Two synthetic jet actuators with acoustic speakers and slot orifices (width $D=1.65 \mathrm{~mm}$, aspect ratio $\alpha=27: 1$ ) are mounted $s=3 D$ apart. The jet-to-surface spacing is stepwise adjustable $(6<H / D<24)$. 


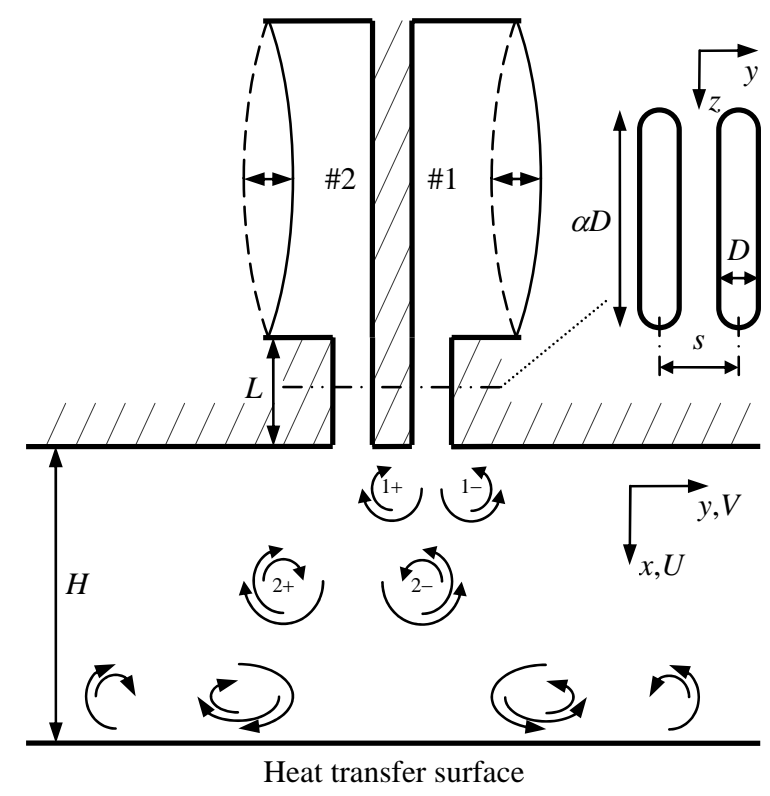

Figure 1. Schematic of the test rig

\subsection{Convective Heat Transfer Coefficient}

The ohmically heated foil (stainless steel AISI 316, $t_{s}=12.5 \mu \mathrm{m}$ thick) is sufficiently thin to be considered a constant heat flux boundary condition. The foil is mounted between two thick copper electrodes. A spring-loaded tensioning mechanism keeps the foil taut. The bottom of the foil is painted matte black. A FLIR ThermoVision ${ }^{\mathrm{TM}} \mathrm{A} 40 \mathrm{M}$ thermal imaging camera measures the temperature distribution $T$ on the bottom of the foil, with a spatial resolution of $2.4 \mathrm{px} / \mathrm{mm}$.

The local convective heat flux $q\left[\mathrm{~W} / \mathrm{m}^{2}\right]$ is determined from the electrical power input $q_{o h m}$, corrected for (i) non-uniform heating, (ii) radiation heat loss $q_{\text {rad }}$ from top and bottom, (iii) convection heat loss $q_{c n v, b}$ from bottom, and (iv) heat spreading $q_{c n d}$ due to lateral conduction within the foil:

$$
q=c_{\text {ohm }} q_{\text {ohm }}-q_{\text {rad }}-q_{c n v, b}-q_{c n d}=h\left(T-T_{j e t}\right)
$$

where $c_{o h m}$ is a local correction for non-uniform heating power due to non-ideal electrical contact between foil and electrodes. This is determined from an initial measurement without flow, and assuming a constant heat transfer coefficient on top of the foil. This local correction varies around 5 to $10 \%$.

The lateral conduction correction is given by $q_{c n d}=k_{s} t_{s} \nabla^{2} T$. Spatial filtering was needed to reduce the propagation of temperature noise through the partial derivatives. Rather than the polynomial fitting approach used by Patil and Narayanan [7] for an axisymmetric geometry, a spatial median filter has been applied with $9 \times 9 \mathrm{px}^{2}$ aperture before and after taking the first order partial derivatives.

The uncertainty in the convective heat transfer coefficient $h=q /\left(T-T_{j e t}\right)$ is given by:

$$
\frac{\Delta h}{h}=\sqrt{\left(\frac{\Delta q}{q}\right)^{2}+\frac{\Delta T^{2}+\Delta T_{j e t}^{2}}{\left(T-T_{j e t}\right)^{2}}}
$$

where the convective heat flux uncertainty $\Delta q / q$ is around $7 \%$. $T_{j e t}$ is measured with a K-type thermocouple, with an estimated uncertainty $\Delta T_{j e t}=0.1{ }^{\circ} \mathrm{C}$. The value of $\Delta T$ results from a combination of uncertainty in the infrared camera measurement and the radiation properties of foil and surroundings. Overall, $\Delta T=0.2^{\circ} \mathrm{C}$.

A determining factor in the overall uncertainty $\Delta h$ is the temperature difference $T-T_{\text {jet }}$. The minimum value is typically around $3{ }^{\circ} \mathrm{C}$, resulting in an uncertainty at the stagnation point of $(\Delta h / h)_{0}=10 \%$. In adverse flow conditions, recirculation of hot air into the jet cavity may cause $T-T_{j e t}$ values below $2{ }^{\circ} \mathrm{C}$, thereby increasing the uncertainty $(\Delta h / h)_{0}$ to $20 \%$. Measurements with excessive uncertainty (>25\%) have been omitted from the results.

\subsection{Synthetic Jet Operating Point}

Smith and Glezer [6] indicate that for a pair of closely spaced synthetic jets, the velocity is influenced by the presence of the adjacent jet, particularly when the jets are driven out of phase. For that reason, they recommend using a pressurevelocity calibration curve, instead of a simpler voltage-velocity curve.

For that reason, a calibration model described by Persoons and O'Donovan [8] is used here to maintain $R e$ and $L_{0}$ at a fixed value, based on the measured cavity pressure (G.R.A.S. 40BH microphone, $0.5 \mathrm{mV} / \mathrm{Pa}$ ). The semi-empirical analytical model is derived from simplified gas dynamics and is valid for the frequency range from zero, over the Helmholtz resonance frequency $f_{0}$ up to a geometry-dependent limit [8]. Model closure is provided by a damping coefficient $K$, which is related to the pressure loss coefficient for steady flow across the orifice. The jet velocity amplitude $U_{m}{ }^{*}$ is obtained from the cavity pressure amplitude $p^{*}$ as follows:

$$
\frac{\rho a U_{m}^{*}}{p^{*}}=\sqrt{\frac{2 V_{c}}{A L^{\prime}}} \cdot \sqrt{\left(\frac{f}{f_{0}}\right)^{2}+{\sqrt{\left(\frac{f}{f_{0}}\right)^{4}+\left(K \frac{V_{c}}{A L^{\prime}} \frac{p^{*}}{\rho a^{2}}\right)^{2}}}^{-1}}
$$

where $L^{\prime}=L+2 \beta D$ is the effective orifice length ( $\left.\beta=2.55\right), K$ $=1.809 \pm 0.15, a$ is the speed of sound, $f_{0}=a /\left(2 \pi L^{\prime}\right)\left(A L^{\prime} / V_{c}\right)^{1 / 2}$ $=343 \mathrm{~Hz}$. For sine wave excitation, $U_{0}=U_{m}{ }^{*} /(\pi / 2)$ which allows to determine $R e$ and $L_{0}$ according to Eq. (1).

\subsection{Flow Field}

Velocity measurements have been performed using particle image velocimetry (PIV). The PIV system comprises a New Wave Solo-II Nd:YAG twin cavity laser $(30 \mathrm{~mJ}, 15 \mathrm{~Hz})$ and a PCO Sensicam ${ }^{\mathrm{TM}}$ thermo-electrically cooled CCD-camera $\left(1280 \times 1024 \mathrm{px}^{2}, 12 \mathrm{bit}\right)$ with $28 \mathrm{~mm}$ lens. A glycol-water aerosol is used for seeding, with a mean particle diameter of $0.25 \mu \mathrm{m}$. Customised optics are used to generate a $0.3 \mathrm{~mm}$ thick light sheet. The CCD-camera is mounted perpendicular to the light sheet. The image magnification is 1:4.1 ( $m=54 \mu \mathrm{m} / \mathrm{px})$. A narrow band pass filter is used with fluorescent paint on the orifice plate to maximise the signal-to-noise ratio near the walls. Phase-locked to the synthetic jet actuator, images are acquired 
for 24 phases per period and 16 vector fields are averaged for each phase. The pulse separation time is determined such that the maximum particle image displacement does not exceed a quarter of the initial interrogation window size. The velocity fields are processed with LaVision DaVis 6.2 software, using multi-grid cross-correlation with window shifting and deformation, and an interrogation window size decreasing from $64 \times 64$ to $16 \times 16 \mathrm{px}^{2}$ at $75 \%$ overlap.

To improve the dynamic velocity range, a multi doubleframe (MDF) PIV technique is applied [9,10]. To this end, images are acquired for two values of the pulse separation time, $\tau=\tau_{\min }=1 / 4 m d_{I} / U_{\max }$ and $\tau=8 \tau_{\min }$. The value $\tau_{\min }$ is optimal for the high velocity jet core region, and an arbitrary choice of $8 \tau_{\min }$ is better suited for the low velocity wall jet region. Persoons et al. $[9,10]$ show a considerable increase in dynamic velocity range by applying the MDF technique, resulting in more accurate velocity vectors in the low velocity wall jet and entrainment regions.

For the present case, Fig. 2 compares the standard PIV approach (Fig. 2a) with a single pulse separation $\tau=\tau_{\min }$ to the MDF-PIV approach (Fig. 2b) with two pulse separation values $\tau=\tau_{\min }$ and $8 \tau_{\min }$. Both use identical multi-grid correlation algorithms and settings described above. Due to the enhanced resolution in the low velocity range, MDF captures the low turbulence levels in the entrainment region (on the right in Fig. 2b) much better than the standard approach does.

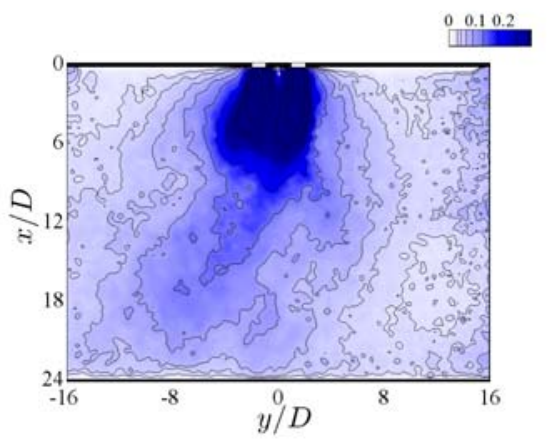

(a)

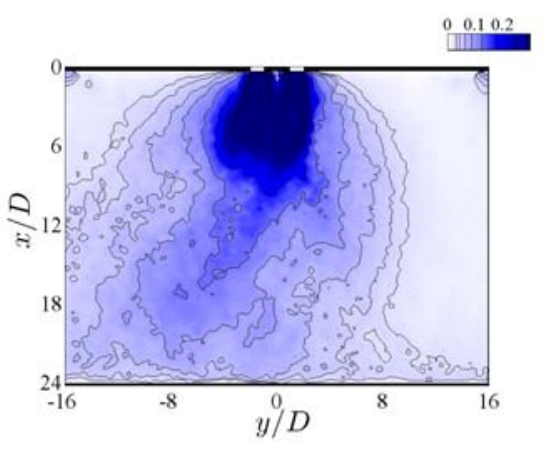

(b)

Figure 2. Effect of applying multi double-frame PIV (a: without MDF, b: with MDF) on the fluctuating wall-normal velocity magnitude $u^{\prime} / U_{0}$ for $L_{0}=29 D, R e=600, H=24 D$ at $\phi=120^{\circ}$.

\section{EXPERIMENTAL RESULTS}

\subsection{Flow Field}

Figure 3 shows selected PIV results (using the MDF approach described above) for an interacting impinging synthetic jet flow, at four values of the phase difference between the jets (top to bottom: $\phi=0^{\circ}, 60^{\circ}, 120^{\circ}, 180^{\circ}$ ). Figures 3a-d show the time-averaged streamlines and velocity magnitude. Figures 3e-h show the fluctuating wall-normal velocity magnitude, since $u$ ' has been related to the local heat transfer coefficient in continuous impinging jets [11]. All velocities are non-dimensionalised with the characteristic velocity $U_{0}$.

The streamline plots in Figs. 3a-d show zero net velocity in the vicinity of the jet orifices, as expected. As the jets are driven progressively out of phase, the jet flow and the stagnation point on the impingement surface shift to the left. This is similar to the vectoring effect for a pair of free synthetic jets [6], where the vectoring direction is toward the side of the jet leading in phase (actuator \#2, on the left in Fig. 1).

Although the jet operating point was identical to Smith and Glezer [6] $\left(L_{0}=29 D, R e=600\right.$ defined as Eq. (1)), the flow patterns at large phase difference are quite different, which is attributable to the confinement effect of the impingement surface. Unlike for free jets, the flow does not attach to the top wall for large $\phi$ values.

Nevertheless, the vectoring effect causes a net cross-flow in the channel, from right to left. This effect is quantified by $\Delta V$, the net transverse velocity, integrated along the channel height, at both edges of the domain:

$$
\Delta V=\left.\frac{1}{H} \int_{0}^{H} V \mathrm{~d} x\right|_{y \rightarrow-\infty}+\left.\frac{1}{H} \int_{0}^{H} V \mathrm{~d} x\right|_{y \rightarrow+\infty}
$$

which means $\Delta V$ is zero for a symmetric flow field, and negative for net cross-flow from right to left. Measured values for $\Delta V$ are listed in Table 1 . For $\phi=0^{\circ}$, small non-zero cross-flow and a flow asymmetry (Fig. 3a) are due to slightly different amplifier characteristics. For $L_{0}=29 D$ and $R e=600$, the cross-flow increases monotonously up to $180^{\circ}$. Other settings $\left(L_{0}=10 \mathrm{D}\right.$, $R e=750$ ) result in a peak cross-flow at a lower $\phi$, as well as significantly different flow patterns (Fig. 4), showing a higher tendency for wall attachment. The influence of the channel height $H$ on $\Delta V$ has not been investigated yet.

Table 1. Magnitudes of cross-flow induced by a pair of interacting impinging synthetic jets at $H=24 D$

\begin{tabular}{rrrrr}
\hline $\boldsymbol{L}_{\mathbf{0}} / \boldsymbol{D}$ & $\boldsymbol{R} \boldsymbol{e}$ & $\boldsymbol{\phi}^{\circ}$ & $\Delta \boldsymbol{V}, \mathbf{m} / \mathbf{s}$ & $\Delta \boldsymbol{V} / \boldsymbol{U}_{\mathbf{0}}$ \\
\hline 29 & 600 & 0 & -0.15 & -0.028 \\
\hline 29 & 600 & 60 & -0.24 & -0.045 \\
\hline 29 & 600 & 120 & -0.55 & -0.103 \\
\hline 29 & 600 & 180 & -0.91 & -0.167 \\
\hline 10 & 750 & 0 & 0.01 & 0.002 \\
\hline 10 & 750 & 60 & -0.66 & -0.097 \\
\hline 10 & 750 & 120 & -1.32 & -0.195 \\
\hline 10 & 750 & 180 & -0.27 & -0.041 \\
\hline
\end{tabular}




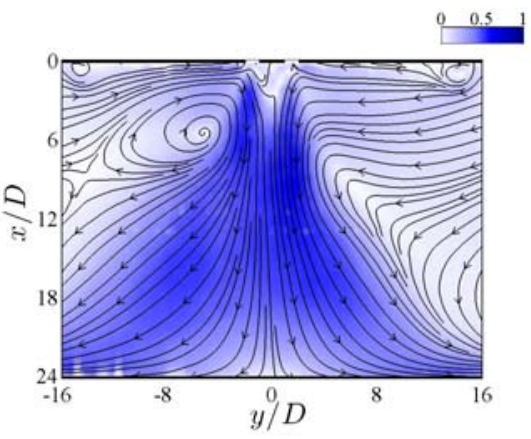

(a)

(e)

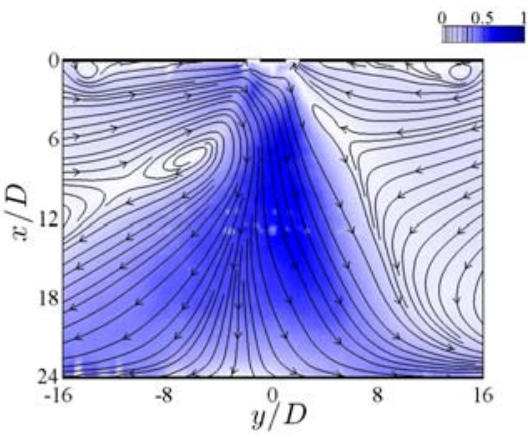

(b)

(f)

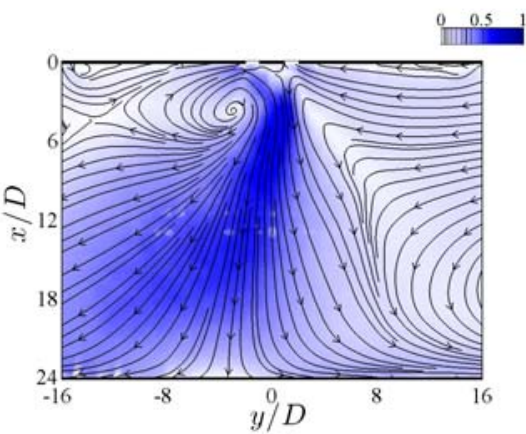

(c)

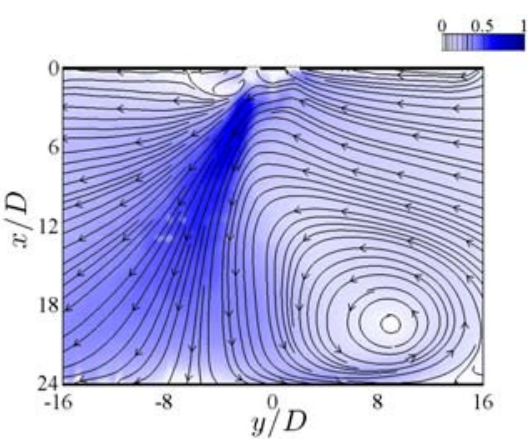

(d)

Figure 3. Flow field for interacting impinging synthetic jets for $L_{0}=29 D, R e=600$, $H=24 D$ : (a-d) time-averaged streamlines and velocity magnitude $\left(U^{2}+V^{2}\right)^{1 / 2} / U_{0},(\mathrm{e}-\mathrm{h})$ fluctuating wall-normal velocity magnitude $u^{\prime} / U_{0}$, at four inter-jet phase differences (a,e) $\phi=0^{\circ},(\mathrm{b}, \mathrm{f}) \phi=60^{\circ},(\mathrm{c}, \mathrm{g}) \phi=120^{\circ},(\mathrm{d}, \mathrm{h}) \phi=180^{\circ}$
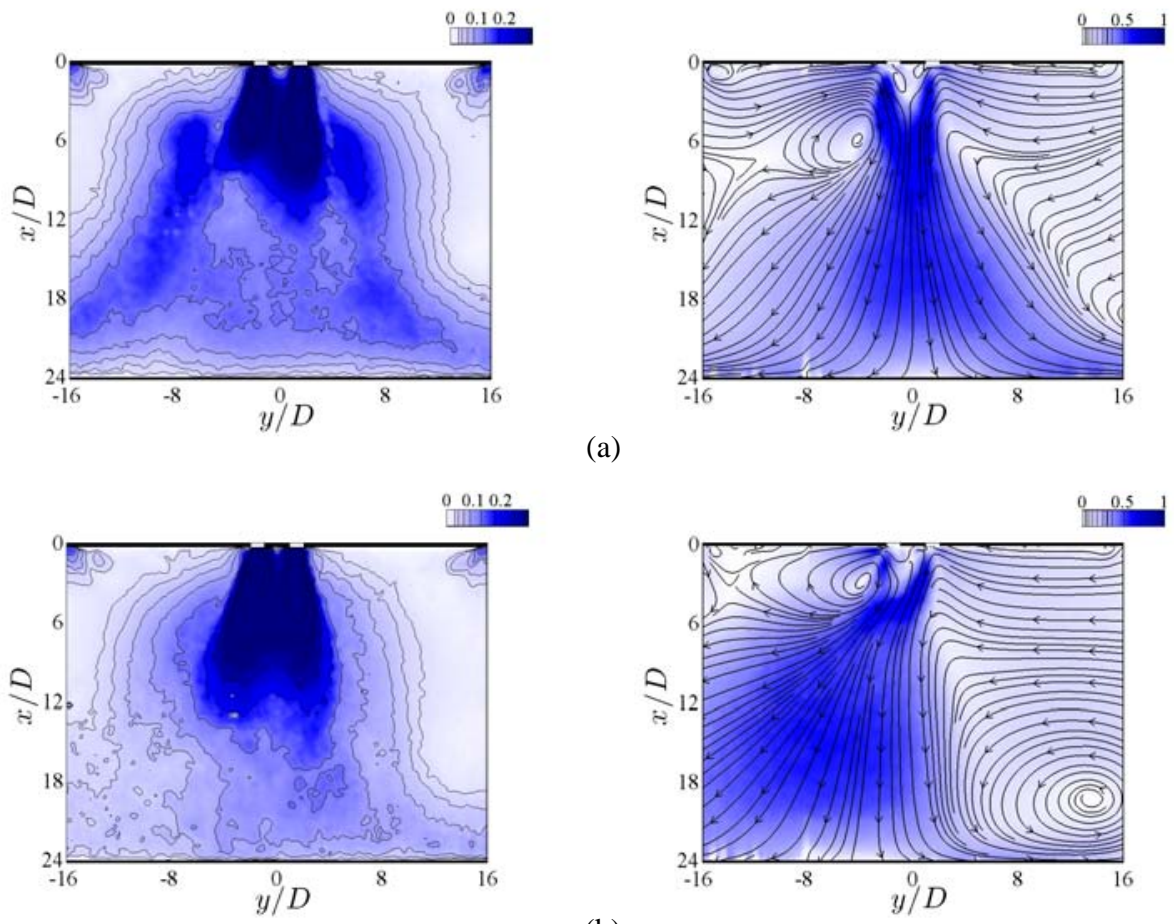

(a)

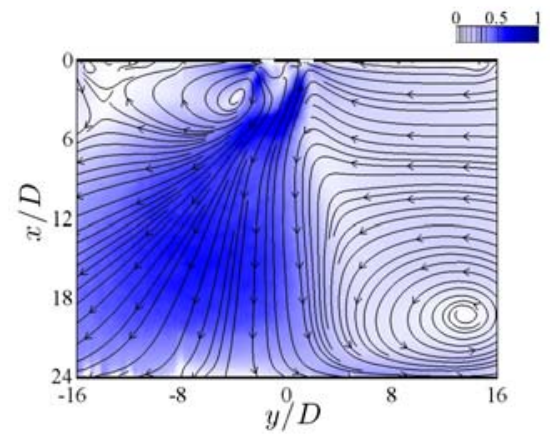

(b)

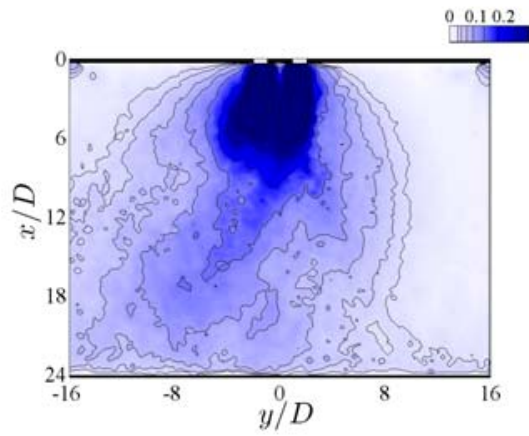

(c)
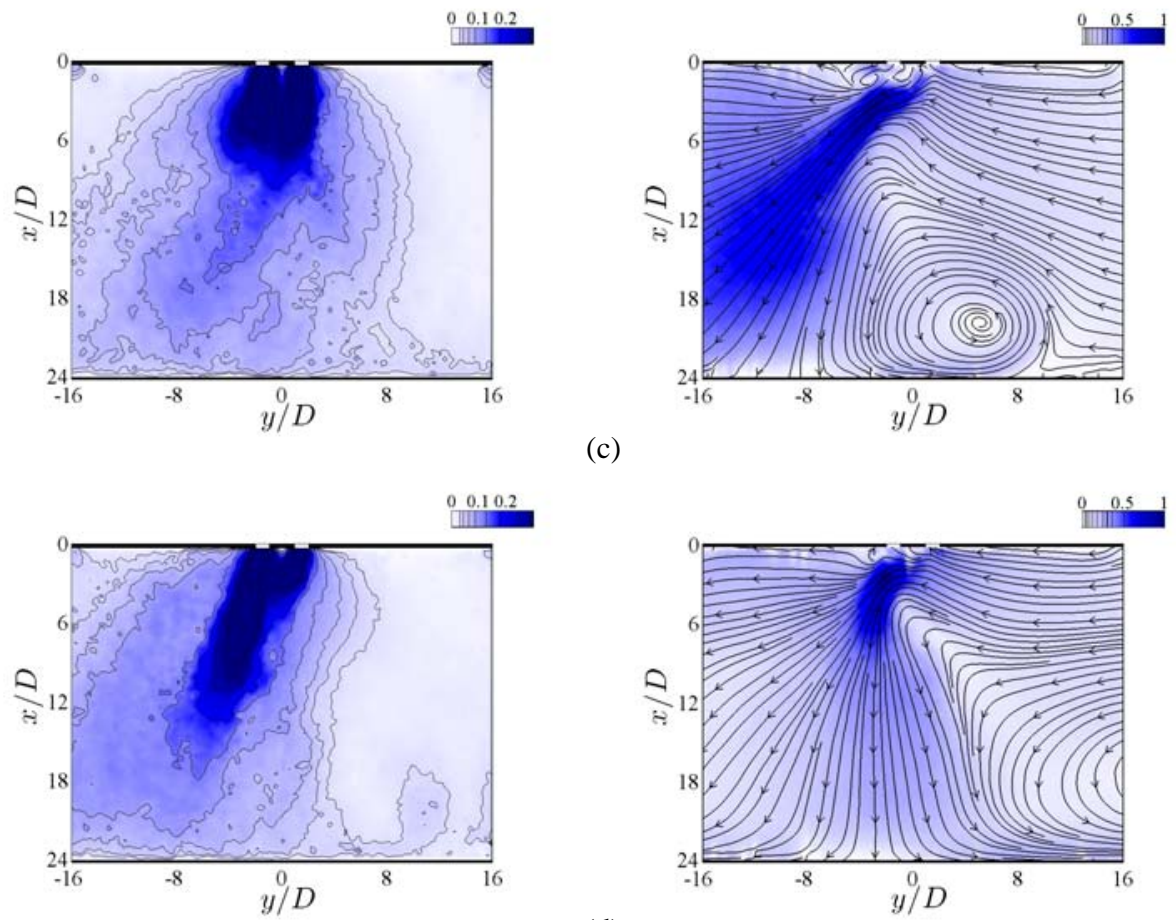

(d)

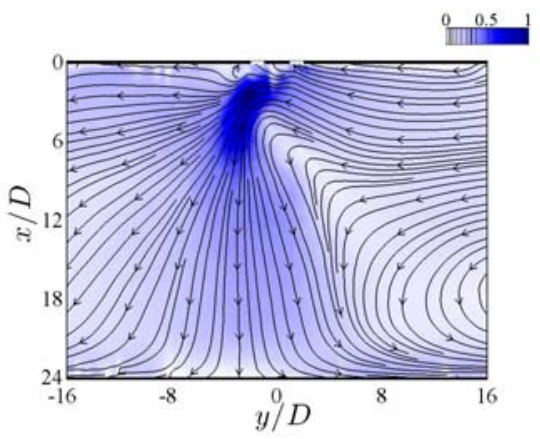

Figure 4. Time-averaged streamlines and velocity magnitude for $L_{0}=10 D, R e=750$, $H=24 D$ at same four inter-jet phase differences 


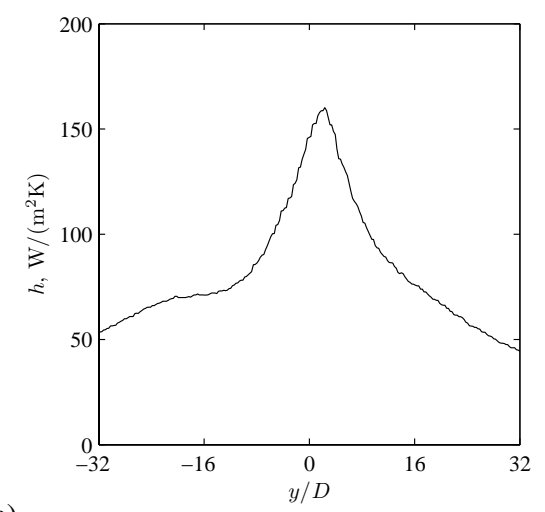

(a)

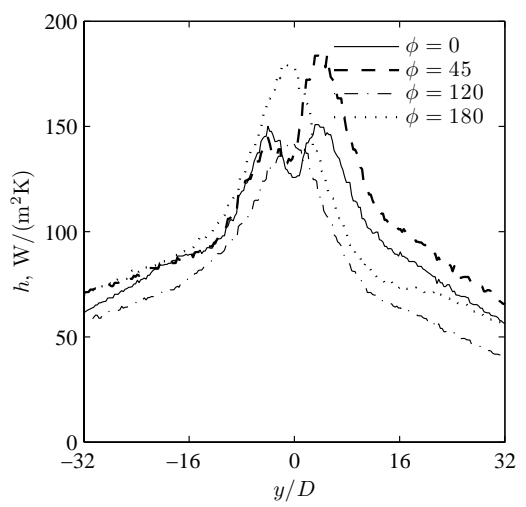

(d)

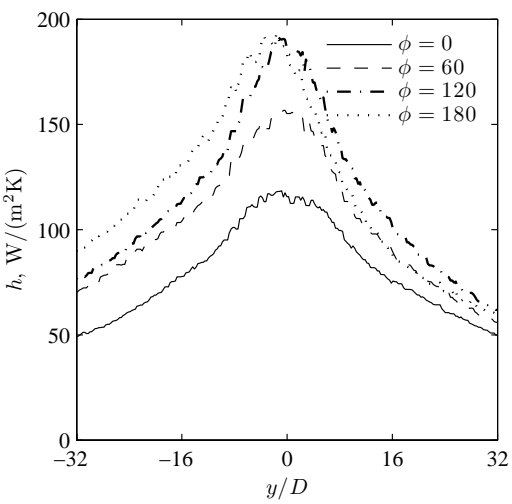

(e)

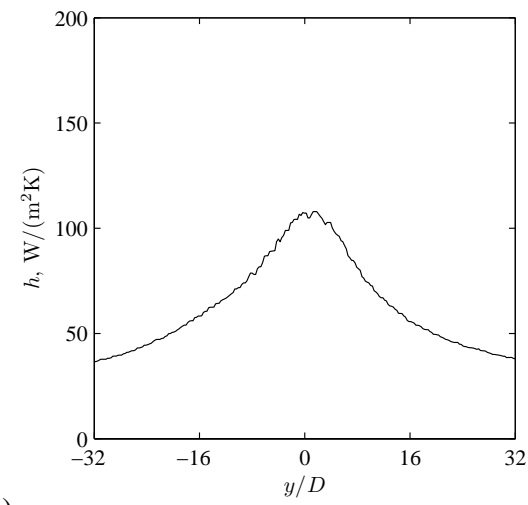

(b)

(c)
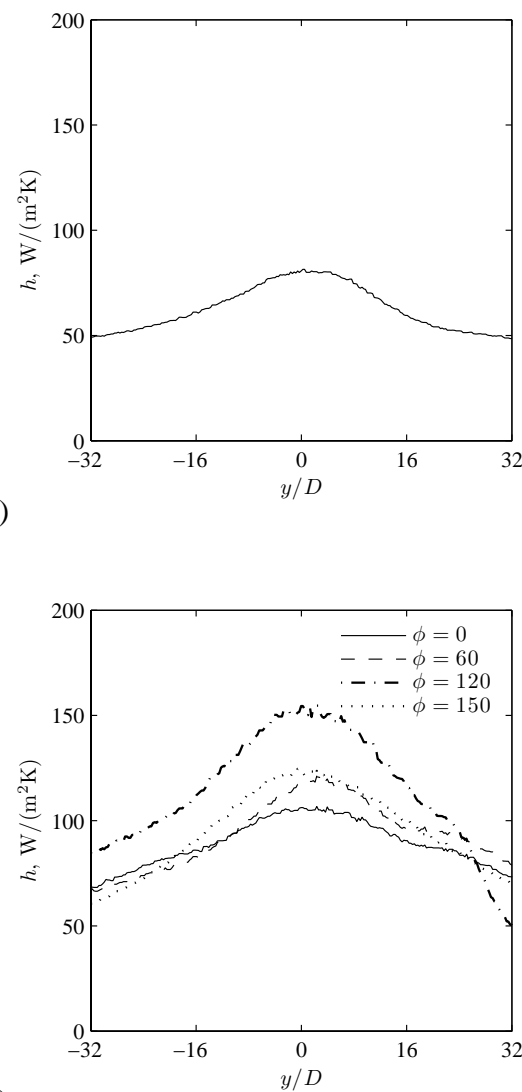

(f)

Figure 5. Profiles of the surface heat transfer coefficient for (a-c) single and (d-f) interacting impinging synthetic jets at $L_{0}=29 D, R e=600$, for three jet-to-surface spacings (a,d) $H=6 D$, (b,e) $H=12 D$, (c,f) $H=24 D$.

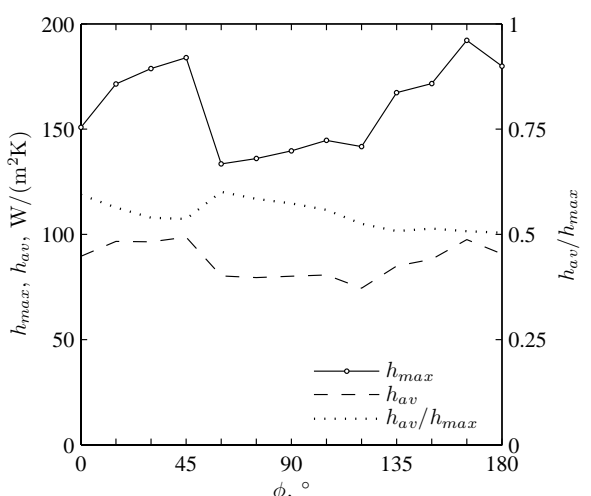

(a)

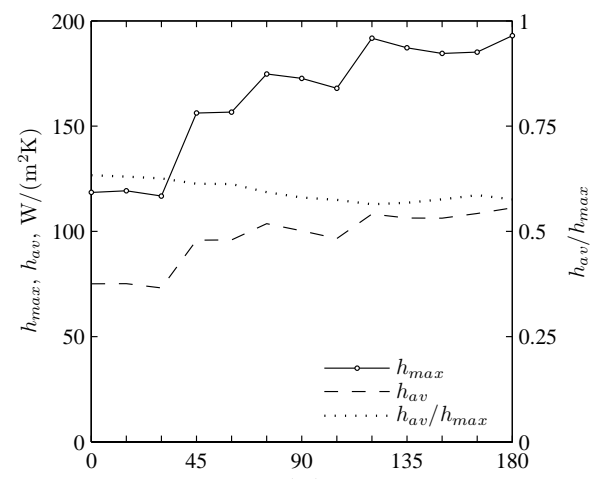

(b)

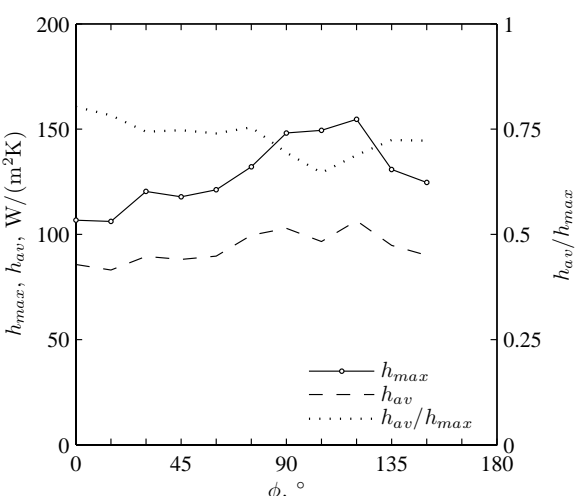

(c)

Figure 6. Phase-dependence of the heat transfer characteristics (peak $h_{\max }$, mean $h_{a v}$, uniformity $h_{a v} / h_{\max }$ ) for interacting impinging synthetic jets at $L_{0}=29 D, R e=600$, for three jet-to-surface spacings (a) $H=6 D$, (b) $H=12 D$, (c) $H=24 D$. 


\subsection{Heat Transfer Profiles}

Figures 5a-c shows the profiles along the $y$ direction of the heat transfer coefficient $h$ for a single jet (\#1 in Fig. 1) operating at $L_{0}=29 D$ and $R e=600$, at three jet-to-surface spacings $H / D=6,12,24$. The asymmetry in Fig. 5a corresponds to the asymmetric flow field in Fig. 3a, which is due to a slight amplifier mismatch. For increasing $H$, the peak heat transfer coefficient $h_{\max }$ decreases and the profile widens.

Figures 5d-f shows the $h$ profiles for interacting jets operated at the same settings for $L_{0}, R e$ and $H$. Each plot shows the profile for four jet phase differences: (-) zero $\phi=0^{\circ}$, () low $\phi=60^{\circ},(--)$ moderate $\phi=120^{\circ},(\cdots \cdots \cdots)$ high $\phi=180^{\circ}$. In some cases, other values are chosen as indicated in Figs. 5d-f.

When comparing the profiles of an in-phase double jet $\left(\phi=0^{\circ}\right)$ to a single jet, the double jet generally features a broader $h$ profile and higher mean heat transfer rate, with one notable exception. At low $H$, the central region experiences a reduction in heat transfer rate because the inner vortices (' $1+$ ' and '2-' in Fig. 1) interact destructively.

As the jets are driven out-of-phase, a different behaviour can be noted for low and high jet-to-surface spacing. Firstly, for high values $(12<H / D<24)$, the heat transfer profile generally increases and shifts slightly to the left, however only in partial agreement with the flow patterns in Figs. 3a-d. The $h$ profile shape becomes asymmetric (increasing more for $y<0$ than for $y>0)$ as $\phi$ increases. Yet the peak heat transfer coefficient remains close to the centre (Fig. $5 f: y \cong 0$ for $\phi=120^{\circ}$ ) and does not follow the stagnation point (Fig. 3c: $y \cong-3.5 D$ for $\phi=120^{\circ}$ ).

Conversely, for low jet-to-surface spacing $(H / D=6)$, the surface is in the formation region of the vortices, as shown by the double-peaked heat transfer profile. As $\phi$ increases $\left(\phi>45^{\circ}\right)$, the overall heat transfer reduces, although a sharp peak remains high due to the proximity of the orifices to the surface. The surface confinement might be hindering the establishment of cross-flow, which in turn would explain the absence of heat transfer enhancement similar to high $H$. Unfortunately no PIV data is available at $H / D=6$ to confirm this.

For continuous impinging jets, O'Donovan and Murray [11] have shown that the local heat transfer coefficient is correlated to the wall-normal velocity fluctuation $u$ ', close to the surface. When examining only the near-wall region, $u$ ' (Figs. 3e-h) provides different information than the mean flow field (Figs. 3a-d). Firstly, the near-wall fluctuation intensity is quite uniform along $y$ for in-phase jets (Fig. 3e: $\phi=0^{\circ}$ ), which seems to agree with the highly uniform heat transfer profile (Fig. 5f). As $\phi$ increases, the near-wall fluctuation intensity reduces, yet an off-centre maximum appears. Interestingly, for $\phi=60^{\circ}$ (Fig. 3f), the peak fluctuation first shifts to the right. For higher values $\phi=120^{\circ}, 180^{\circ}$ (Figs. 3g-h), the peak shifts to the left corresponding to the jet vectoring side. This behaviour is mirrored to some extent in the heat transfer profiles (Fig. 5f), showing an increase towards $y>0$ for $\phi=60^{\circ}$ and an increase towards $y<0$ for $\phi=120^{\circ}$.

However, the heat profile behaviour is likely influenced by the fluctuation intensity as well as the introduction of fresh air due to the cross-flow. As such, the air temperature field would bring more insight into the underlying heat transfer mechanisms.

\subsection{Heat Transfer Characteristics}

Three measures are defined to characterise the heat transfer profiles:

(i) The peak heat transfer coefficient $h_{\max }$, which cannot strictly be called 'stagnation' value since the experiments have shown that the stagnation point does not correspond to the location of maximum heat transfer.

(ii) The averaged heat transfer coefficient, $h_{a v}=\int_{-32 D}^{+32 D} h \mathrm{~d} y$, neglecting any variations in $z$ direction. For this orifice shape (aspect ratio $\alpha=27: 1$ ), the cooling effect extends in $z$ direction over $\Delta \mathrm{z}>1.7 \alpha \mathrm{D}$ for $6<H / D<24$.

(iii) A uniformity measure, $h_{m} / h_{\max }$, which tends to unity for an profile with constant $h$ value.

These measures are used to quantify the cooling performance as a function of jet phase difference $\phi$ and jet-to-surface spacing:

Figure 6 shows the evolution of these quantities as a function of $\phi$, for $6<H / D<24$. For high jet-to-surface spacing $(12<H / D<24)$, the peak value $h_{\max }$ attains a maximum around $\phi=120^{\circ}$. The maximum value $h_{\max }\left(120^{\circ}\right)$ varies between 1.4 and 1.6 times $h_{\max }\left(0^{\circ}\right)$, and 1.7 to 1.9 times $h_{\max }$ for a single jet.

The averaged heat transfer coefficient $h_{a v}$ shows a similar phase-dependence, albeit less pronounced. As a result, the uniformity $h_{m} / h_{\max }$ is relatively independent of $\phi$, yet increases with jet-to-surface spacing.

Table 2 lists values for significant cases, corresponding to Figs. 5 and 6. The final column gives the enhancement ratio of average heat transfer for the double jet versus the corresponding single jet case.

Table 2. Heat transfer characteristics for single and a pair of interacting impinging synthetic jets at $L_{0}=29 D$ and $R e=600$

\begin{tabular}{rrrrrr}
\hline $\boldsymbol{H} / \boldsymbol{D}$ & jets & $\boldsymbol{\phi}^{\circ}$ & $\begin{array}{r}\boldsymbol{h}_{\mathbf{m a x}}, \\
\mathbf{W} /\left(\mathbf{m}^{\mathbf{2}} \mathbf{K}\right)\end{array}$ & $\mathbf{W} /\left(\mathbf{m}^{2} \mathbf{K}\right)$ & $\boldsymbol{h}_{\boldsymbol{a v}} \mathbf{h}_{\boldsymbol{a v}, \mathbf{1}}$ \\
\hline 6 & 1 & - & 160 & 77 & - \\
\hline 6 & 2 & 0 & 151 & 90 & 1.17 \\
\hline 6 & 2 & 45 & 184 & 100 & 1.29 \\
\hline 6 & 2 & 120 & 142 & 74 & 0.97 \\
\hline 6 & 2 & 180 & 180 & 91 & 1.18 \\
\hline 12 & 1 & - & 108 & 59 & - \\
\hline 12 & 2 & 0 & 119 & 75 & 1.28 \\
\hline 12 & 2 & 60 & 157 & 96 & 1.63 \\
\hline $\mathbf{1 2}$ & $\mathbf{2}$ & $\mathbf{1 2 0}$ & $\mathbf{1 9 2}$ & $\mathbf{1 0 8}$ & $\mathbf{1 . 8 4}$ \\
\hline $\mathbf{1 2}$ & $\mathbf{2}$ & $\mathbf{1 8 0}$ & $\mathbf{1 9 3}$ & $\mathbf{1 1 1}$ & $\mathbf{1 . 8 9}$ \\
\hline 24 & 1 & - & 81 & 60 & - \\
\hline 24 & 2 & 0 & 107 & 86 & 1.42 \\
\hline 24 & 2 & 60 & 121 & 90 & 1.49 \\
\hline $\mathbf{2 4}$ & $\mathbf{2}$ & $\mathbf{1 2 0}$ & $\mathbf{1 5 5}$ & $\mathbf{1 0 7}$ & $\mathbf{1 . 7 7}$ \\
\hline 24 & 2 & 150 & 125 & 90 & 1.49 \\
\hline
\end{tabular}




\section{DISCUSSION: OPTIMAL PERFORMANCE}

The paper has shown flow field and heat transfer results as a function of $\phi$ and $H$. For $\phi=0^{\circ}$, the heat transfer distribution resembles that of a single jet, since the inner vortices cancel out, at least for sufficiently high jet-to-surface spacing $(H>12 D)$. When driven progressively out of phase, the flow is vectored to the left side $(y<0)$ towards the phase-leading jet. A different behaviour can be noted for the mean flow and fluctuation intensity, which can partly explain the heat transfer behaviour.

At a small phase difference $\left(60^{\circ}<\phi<120^{\circ}\right)$, this enhances the heat transfer as the induced cross-flow draws in fresh air, yet the vortical flow still impinges the surface quite strongly. This is demonstrated by the high fluctuation intensity near the centre (Fig. 3g).

At a large phase difference $\left(120^{\circ}<\phi<180^{\circ}\right)$, the heat transfer levels off or decreases since the vortices travel further and dissipate more before impingement, although the resulting cross-flow may be stronger.

An optimal phase difference of $\phi \cong 120^{\circ}$ can be identified, although this value is $H$-dependent. The optimum phase decreases for increasing jet-to-surface spacing, since the distance travelled by the vortices determines their degree of dissipation, and this scales with $H$.

For a small jet-to-surface spacing $(H=6 D)$, the heat transfer does not show a similar increase as a function of $\phi$, likely due to the proximity of the surface which seems to inhibit cross-flow establishment.

As a result, the mid value of $H=12 D$ (in combination with $\phi \cong 120^{\circ}$ ) results in the best cooling performance. Since the $\phi$ dependence for peak and average heat transfer is quite similar, the optimum phase maximizes both local and average heat transfer rates.

\section{CONCLUSION}

This study has investigated the convective cooling performance of two adjacent interacting impinging synthetic jets. Infrared thermography and particle image velocimetry have been used to determine the surface heat transfer distribution and the flow field, respectively.

A multi double-frame PIV technique has increased the dynamic velocity range, thereby enhancing the PIV vector quality in low velocity regions such as the wall jet and the entrainment region.

The cooling performance has been quantified in terms of the maximum, average and uniformity of the heat transfer coefficient distribution.

The current study is carried out for a fixed jet geometry (slot jets with aspect ratio $\alpha=27: 1$, with separation distance $s=3 D$ ), fixed stroke length $L_{0}=29 D$ and Reynolds number $R e=600$. The performance is optimised for the phase difference between the jets $\phi$ and the jet-to-surface spacing $H$. Both maximum and average cooling rates show an optimum around $\phi \cong 120^{\circ}$, although this value is also slightly dependent of $H$.
In this optimum configuration, the interacting synthetic jet yields an enhancement of about $90 \%$ in average heat transfer rate compared to the corresponding single jet (see Table 2), without needing an external cross-flow device.

As a part of ongoing research, the optimization will be expanded to include other relevant parameters. In first instance the effect of stroke length $L_{0}$ will be looked at, since for single round synthetic jets, the heat transfer characteristics have been found to primarily depend on $L_{0} / H$ [5].

\section{ACKNOWLEDGMENTS}

Dr. Tim Persoons is a postdoctoral research fellow of the Irish Research Council for Science, Engineering and Technology (IRCSET). The authors also acknowledge the financial support of Science Foundation Ireland (Grant no. 07/RFP/ENM123). This work is performed in the framework of the Centre for Telecommunications Value-Chain Research (CTVR).

\section{NOMENCLATURE}

$\begin{array}{ll}A & \text { Jet orifice cross-sectional area, } \mathrm{m}^{2} \\ a & \text { Speed of sound, } \mathrm{m} / \mathrm{s} \\ D & \text { Jet orifice width, } \mathrm{m} \\ d_{I} & \text { PIV interrogation window size, } \mathrm{px} \\ f, f_{0} & \text { Jet actuation and Helmholtz resonance frequency, } \mathrm{Hz} \\ H & \text { Jet to surface spacing, } \mathrm{m} \\ h & \text { Local convective heat transfer coefficient, based on } \\ & T_{\text {jet }}, \mathrm{W} /\left(\mathrm{m}^{2} \mathrm{~K}\right) \\ h_{0}, h_{m} & \text { Peak and mean heat transfer coefficient, } \mathrm{W} /\left(\mathrm{m}^{2} \mathrm{~K}\right) \\ k, k_{s} & \text { Thermal conductivity of air and foil, } \mathrm{W} /(\mathrm{mK}) \\ K & \text { Jet orifice damping coefficient } \\ L, L & \text { Jet orifice length (geometric and effective), } \mathrm{m} \\ L_{0} & \text { Synthetic jet stroke length }\left(L_{0}=2 U_{0} / f\right), \mathrm{m} \\ m & \text { PIV image pixel resolution, } \mathrm{m} / \mathrm{px} \\ N u & \text { Nusselt number }(N u=h D / k) \\ p & \text { Relative jet cavity pressure, Pa } \\ q & \text { Convective heat flux, } \mathrm{W} / \mathrm{m}^{2} \\ R e & \text { Reynolds number }\left(R e=\rho U_{0} D / \mu\right) \\ s & \text { Jet centre separation distance, } \mathrm{m} \\ T, T_{j e t} & \text { Heated surface and jet cavity temperature, }{ }^{\circ} \mathrm{C} \\ t & \text { Time, } \mathrm{s} \\ t_{s} & \text { Heated foil thickness, } \mathrm{m} \\ U_{0} & \text { Average orifice velocity during ejection, } \mathrm{m} / \mathrm{s} \\ U, V & \text { Wall-normal (vertical) and transverse velocity, } \mathrm{m} / \mathrm{s} \\ u, v & \text { Wall-normal (vertical) and transverse fluctuating } \\ & \text { velocity magnitude, } \mathrm{m} / \mathrm{s} \\ V_{c} & \text { Jet cavity volume, }{ }^{3} \\ x, y & \text { Wall-normal (vertical) and transverse coordinate, } \mathrm{m}\end{array}$

\section{Greek symbols}

$\alpha \quad$ Jet orifice aspect ratio (span to width)

$\phi \quad$ Phase difference between synthetic jet actuators, ${ }^{\circ}$

$\mu \quad$ Dynamic viscosity of air, Pa.s

$\rho \quad$ Density of air, $\mathrm{kg} / \mathrm{m}^{3}$ 


\section{REFERENCES}

1 Gillespie, M. B., Black, W. Z., Rinehart, C., and Glezer, A., 2006, "Local convective heat transfer from a constant heat flux flat plate cooled by synthetic air jets," ASME J. Heat Transfer, 128(10):990-1000.

2 Pavlova, A., and Amitay, M., 2006, "Electronic cooling using synthetic jet impingement," ASME J. Heat Transfer, 128(9):897-907.

3 Campbell, J. S., Black, W. Z., Glezer, A., and Hartley, J. G., 1998, "Thermal management of a laptop computer with synthetic air microjets,” Proc. IEEE Intersociety Conference on Thermal and Thermomechanical Phenomenon in Electronic Systems, pp. 43-50.

4 Smith, B. L., and Glezer, A., 1998, "The formation and evolution of synthetic jets,” Phys. Fluids, 10(9):2281-2297.

5 Valiorgue, P., Persoons, T., McGuinn, A., and Murray, D. B., 2009, "Heat transfer mechanisms in an impinging synthetic jet for a small jet-to-surface spacing," Exp. Therm. Fluid Sci., in press.

6 Smith, B. L., and Glezer, A., 2005, "Vectoring of adjacent synthetic jets," AIAA J., 43(10):2117-2124.

7 Patil, V. A., and Narayanan, V., 2005, “Application of heated-thin-foil thermography technique to external convective microscale flows," Meas. Sci. Technol., 16(2):472-476.

8 Persoons, T., and O’Donovan, T. S., 2007, “A pressure-based estimate of synthetic jet velocity," Phys. Fluids, 19(12):128104.

9 Persoons, T., O’Donovan, T. S., and Murray, D. B., 2008, "Improving the measurement accuracy of PIV in a synthetic jet flow,” Int. Symp. Appl. Laser Techn. Fluid Mech., Lisbon, Portugal, 7-10 July.

10 Persoons, T., and O’Donovan, T. S., 2009, "Multi doubleframe particle image velocimetry," Meas. Sci. Technol., in review.

11 O’Donovan, T. S., and Murray, D. B., 2007, “Jet impingement heat transfer - Part II: A temporal investigation of heat transfer and local fluid velocities,” Int. J. Heat Mass Transfer, 50(17-18):3302-3314. 